Article

\title{
Study on Rheological Behavior of Micro/Nano-Silicon Carbide Particles in Ethanol by Selecting Efficient Dispersants
}

\author{
Guoqiang Luo, Zhuang Zhang, Jianian Hu, Jian Zhang ${ }^{\mathbb{D}}$, Yi Sun *, Qiang Shen \\ and Lianmeng Zhang
}

State Key Laboratory of Advanced Technology for Materials Synthesis and Processing, Wuhan University of Technology, Wuhan 430070, China; luogq@whut.edu.cn (G.L.); zz1994@whut.edu.cn (Z.Z.); hujianian1@outlook.com (J.H.); zhangjian178@whut.edu.cn (J.Z.); sqqf@263.net (Q.S.); lmzhang@whut.edu.cn (L.Z.)

* Correspondence: sunyiwhut@163.com; Tel.: +86-1877-102-1416

Received: 8 February 2020; Accepted: 16 March 2020; Published: 25 March 2020

check for updates

\begin{abstract}
A colloidal stability study of a nonaqueous silicon carbide suspension is of great significance for preparing special silicon carbide ceramics by colloidal processing. In this paper, three different chemical dispersants, which are amphiphilic, acidophilic, and alkaliphilic, are selected to compare their ability to stabilize nonaqueous slurries of silicon carbide. The analysis of the flow index factor is first used to estimate the colloidal stability of the suspensions. The results show that the addition of only $5 \mathrm{wt}$.\% polyvinylpyrrolidone (PVP) forms a silicon carbide slurry with a low viscosity value of $17 \mathrm{mPa} \cdot \mathrm{s}$ at $25 \mathrm{~s}^{-1}$. In addition, Fourier transform infrared spectroscopy (FTIR) and X-ray photoelectron spectroscopy (XPS)measurements indicate that the PVP molecule is successfully adsorbed on the surface of silicon carbide. The different adsorption models are fitted, and the adsorption of PVP molecules on the surface of silicon carbide belongs to the Langmuir single-layer adsorption model. At the optimal PVP amount, the volume content of the suspension is as high as 22.27 vol.\%, a Newtonian-like fluid still appears, and no agglomerate structure is formed in the system. After the volume content exceeds 22.27 vol.\%, the flow index factor of the slurry begins to plummet, indicating that the slurry begins to transform from a Newtonian-like fluid to a shear-thinning fluid. The particles undergo inevitable agglomeration accompanied by the emergence of yield stress. Finally, a maximum solid loading of the system is predicted to be 46 vol. $\%$, using the Krieger-Dougherty model.
\end{abstract}

Keywords: rheology; SiC suspension; colloidal stability; dispersion mechanism; flow index factor

\section{Introduction}

Silicon carbide ceramics are emerging candidates for special ceramics owing to their high hardness, high mechanical strength, high antioxidant capacity, and corrosion resistance. Silicon carbide ceramics are widely used in the armor, aerospace, medical, chemical, steel metallurgy, and electronics industries, as well as other fields. However, owing to the high strength and high brittleness of silicon carbide $(\mathrm{SiC})$, the preparation of silicon carbide devices with complex sizes is limited, which increases the economic cost in their subsequent processing [1-5].

As a result, increasing attention has been focused on colloidal processing methods, which can lead to complex shapes and uniform microstructures of green bodies. In recent years, colloidal processing, such as slip casting and tape casting, has been widely used to produce special ceramics from SiC powders [6-9]. Therefore, it is particularly important to obtain ceramic suspensions with good dispersion 
stability and reliable rheological behaviors. The colloidal techniques first need to achieve sufficient dispersion of the ceramic particles in the solvent. In particular, a starting suspension with high ceramic loading and low viscosity is also required [10-12].

The scale effect of nanopowders offers various advantages, and these powders have attracted widespread attention for their selection as raw powders for molding. However, micro- and nanopowders, owing to their high surface energy, are found to form agglomerated structures in solvents extremely easily, which affects the subsequent processing $[13,14]$. When using water as a solvent, owing to a large number of hydrogen bonds in the water, additional hydrogen bonds are easily formed between the particles and cause agglomeration. Additionally, the surface tension of water is so high that it is difficult to wet the fine powder, which limits its application in a colloidal process. In contrast, organic solvents have low surface tension, so environmentally-friendly organic solvents have attracted more attention [15-18].

At present, a large number of studies have focused on achieving good dispersion of silicon carbide powder into water, and the commonly used dispersants are mainly small molecules and polyelectrolytes, such as tetramethylammonium hydroxide (TMAH) and polyethyleneimine (PEI) [19-30]. For our own research, tape casting is used to obtain dense $\mathrm{SiC}$ ceramics. In terms of solvent options, we chose ethanol as the solvent because, to date, it is difficult to find a suitable binder that can be efficiently dissolved into water; additionally, the colloidal process usually requires the addition of a variety of polymers, which are prone to produce complex effects in water, and we lack certain knowledge about what those interactions are [19-25,28-32]. Unfortunately, most of these studies were performed in deionized water. Only a few studies have focused on stabilization mechanisms in organic solvents [26,27]. Prasenjit et al. [33] reported a few parameters on the rheological behavior of SiC nanoparticles in an aqueous solvent. However, for the rheological analysis, only the viscosity data of the suspension are analyzed, and no further analysis is made on the change of the fluid form. Moreover, there is no study on the maximum solids content of the suspension, which is an important parameter in practical applications.

Polyvinylpyrrolidone (PVP) has been widely used in current markets. PVP was originally applied as an extension agent for plasma substitutes and was later widely used in diverse fields. PVP is an amphiphilic polymer that is harmless to humans and the environment [34-36]. For common organic solvents, such as amines, alcohols, and acids, PVP easily dissolves into them. The polymer also has a long history in the food industry and ceramic processing as a highly efficient multifunctional dispersant.

PEI is an effective dispersant in a low $\mathrm{pH}$ suspension. The stability mechanism of PEI in suspension is a combination of steric hindrance and surface charge repulsion mechanisms that has been successfully applied to stabilize different ceramic suspensions [19-24]. The ability of PEI to acquire hydrogen ions was affected by the $\mathrm{pH}$ value; the protonation $(\sim 0.1 \%)$ of PEI almost occurred at $\mathrm{pH} 10$, and it was completely protonated at $\mathrm{pH}$ 2. PEI is a polyelectrolyte with many branches. During the polymerization process, the polymerization of aziride leads to the formation of PEI, some oligomers are produced, and many tertiary amine groups are present, causing PEI to have a high charge density. This also makes it acidophilic. The high branching properties of PEI allow for a high charge concentration and various molecular weights; therefore, it can also act as a chain-entangled coagulation agent to agglomerate the particles [37].

Tetramethylammonium hydroxide (TMAH, 25\% aqueous solution, $91.153 \mathrm{~g} / \mathrm{mol}$, Sigma-Aldrich $\mathrm{Co}$, Shanghai, China) has a chemical formula of $\mathrm{N}(\mathrm{CH} 3)_{4}{ }^{+} \mathrm{OH}^{-}$and is a quaternary ammonium salt. Its stabilizing mechanism is mainly its use as a free electrolyte, which increases the ionic strength and disturbs the electrostatic repulsion between particles; this causes a bridging effect between particles and leads to slight agglomerations [25].

In the present work, the effects of different dispersants, dispersant concentrations, and solids loading on the rheological properties of silicon carbide nonaqueous slurries were investigated. When PVP was used as a dispersing agent, an organic suspension of well-dispersed silicon carbide was prepared. The purpose of this work was to use rheological knowledge to quantitatively 
evaluate the efficiency of dispersant. The maximum solid loading was estimated by the Einstein and Krieger-Dougherty models. The adsorption mechanism was determined using classical monolayer Langmuir isotherm and multilayer Freundlich isotherm models, and the possible mechanism of dispersant stabilization was also investigated.

\section{Materials and Methods}

\subsection{Starting Materials}

The powder used was silicon carbide (Aladdin, Shanghai, China, $>99 \%$ purity), and the average particle size was $303 \mathrm{~nm}$ (d50). The average size (d50) of the powder was determined by laser diffraction (Mastersizer 2000, Wuhan University of Technology, Wuhan, China). The crystal structure of raw material powder was identified by X-ray diffraction (XRD) using $\mathrm{Cu} \mathrm{K} \alpha$ radiation. (RU-200B/D/MAX-RB, Rigaku, Tokyo Japan). Scanning electron microscopy (SEM, Zeiss Ultra Plus/Zeiss Ultra Plus, Carl Zeiss AG, Shanghai, China) was used to investigate the morphology and size of silicon carbide powders. The technical characteristics of silicon carbide surface chemistry were determined by X-ray photoelectron spectroscopy (XPS) (ESCALAB 250Xi, Thermo Fisher Scientific, Shanghai, China) and Fourier transform infrared spectroscopy (FTIR) (Nicolet 6700, Thermo Electron Scientific Instruments, Shanghai, China). The dispersants used include PVP (Mv 50,000, >99\% Shanghai Aladdin Biochemical Technology Co., Ltd, Shanghai, China), polymeric polyethyleneimine (PEI) with molecular weights of $1800 \mathrm{~g} / \mathrm{mol}$ (Aladdin Industrial Corporation, Shanghai, China), and TMAH (Mv 91.15, 25\% aqueous solution, Aladdin Industrial Corporation, Shanghai, China). The structural details of the three dispersants are shown in Figure 1, and the spatial structures were optimized in an Advanced Chemistry Development (ACD) /3D Viewer. An organic solvent of ethanol ( $\geq 99.7 \%)$ was used as a dispersion medium.

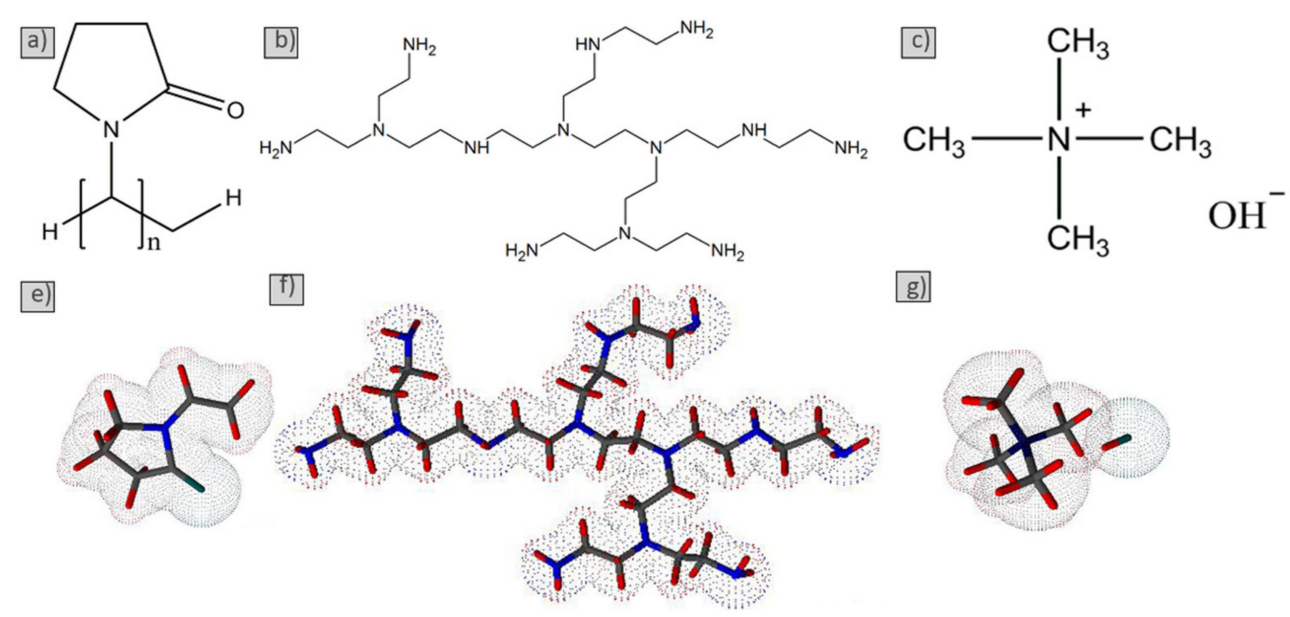

Figure 1. Chemical and partial spatial formula of polyvinylpyrrolidone (PVP) (a,e), polyethyleneimine (PEI) $(\mathbf{b}, \mathbf{f})$, and tetramethylammonium hydroxide (TMAH) $(\mathbf{c}, \mathbf{g})$.

\subsection{Preparation of SiC Suspensions}

The silicon carbide powder was mixed with ethanol, and then dispersant was added. Each dispersant content was from $1 \mathrm{wt} . \%$ to $6 \mathrm{wt}$ \% (based on a certain amount of the powder weight), and solids loading of the ceramic suspensions was fixed at $50 \mathrm{wt} \%$ (20 vol. $\%$ ). The volume fractions of the suspension were calculated using Equation (1):

$$
\varphi_{v}=\frac{m_{n} / \rho_{n}}{\left(m_{n} / \rho_{n}\right)+\left(m_{b} / \rho_{b}\right)}
$$


where $\varphi_{v}$ denotes the volume fraction of suspension (\%); $m_{n}$ and $m_{b}$ are the weight of nanoparticles and the base medium, respectively; and $\rho_{n}$ and $\rho_{b}$ determine the densities of the nanoparticle and base liquid, respectively.

In a practical process, ball milling containing $5 \mathrm{~mm}$ yttrium oxide-stabilized zirconia balls (QM-QX0.4, Nanjing Nanda Instrument Co. Ltd, Nanjing, China) was used to mix and deagglomerate suspensions at $300 \mathrm{rpm}$ for $24 \mathrm{~h}$ at room temperature. Then, suspensions were evaluated with respect to their rheology by means of viscosity measurements (Brookfield, Model LVDV-II+P, Shanghai, China).

\subsection{Rheological Measurement}

A concentric cylinder viscometer was applied to measure the rheological properties of the $\mathrm{SiC}$ dispersion (Brookfield, Model LVDV-II+P). A thermostatic system was used along with the experiment, and the temperature was kept at $21^{\circ} \mathrm{C}$. In the flow test, the shear rates ranged from 0 to $200 \mathrm{~s}^{-1}$ with 50 step intervals and a wait of $2 \mathrm{~s}$ before each shear rate change. All rheological measurements were pre-sheared for $1 \mathrm{~min}$ at $100 \mathrm{~s}^{-1}$ followed by $30 \mathrm{~s}$ at a $0 \mathrm{~s}^{-1}$ shear rate to eliminate any effects owing to the loading of samples.

\subsection{Adsorption Equilibrium of Dispersants on SiC Powder}

The adsorption performance of PVP was investigated by the following method: a suspension having a solids content of $20 \mathrm{vol} \%$ with a dispersant of different concentrations (according to the weight of the powder) was centrifuged at $9000 \mathrm{rpm}$ to obtain $\mathrm{SiC}$ particles from the suspension. The obtained powder was dried in a thermostatic blast oven at $100^{\circ} \mathrm{C}$, and the weight of the adsorbed PVP on the particles was measured via a thermogravimetric technique (Q50, TA Instruments Ltd., Crawley, UK).

\subsection{FTIR and XPS Measurements}

The interaction of the dispersant on the surface of SiC particles was studied in a $400-4000 \mathrm{~cm}^{-1}$ region by Fourier transform infrared spectroscopy (Nicolet 6700, Thermo Electron Scientific Instruments, Shanghai, China). The powder was placed in a sample box, which was then placed in a diffuse reflection accessory. To improve the signal-to-noise ratio, the angle of the mirror was adjusted. The infrared spectroscopy of the silicon carbide powder that adsorbed PVP was collected using 128 scans with a background of pure silicon carbide powder at a resolution of $2 \mathrm{~cm}^{-1}$. In addition, the chemical properties of the silicon carbide particle surface were measured by XPS (ESCALAB 250Xi, Thermo Fisher Scientific, Shanghai, China).

\section{Results and Discussion}

\subsection{Characterization of the Silicon Carbide Powder}

The XRD peak (Figure 2) of the silicon carbide powder confirmed the existence of only the $\beta-\mathrm{SiC}$ cubic sphalerite crystal structure, and PVP modified method does not alter the crystal structure of $\mathrm{SiC}$. The particle size measurement and SEM results of the initial SiC particles are shown in Figure 3. The size distribution of $\mathrm{SiC}$ powder was distinctly bimodal, with a slender peak at $0.1 \mu \mathrm{m}$ and a broad peak at $1 \mu \mathrm{m}$. The particle size was $0.303 \mu \mathrm{m}$ at D50, and the particle size value provided by the manufacturer was $0.5 \mu \mathrm{m}$; multiple size analyses were conducted to confirm data accuracy and repeatability. From the graph above, we can substantially see that no particles having a size beyond these two ranges were detected. The SEM results were basically consistent with the particle size data; most of the particles were $\sim 1 \mu \mathrm{m}$, and the fines were easily observed. Scanning electron microscopy (SEM) images also showed that the morphology of these particles was irregular and "plate-like" in nature. 


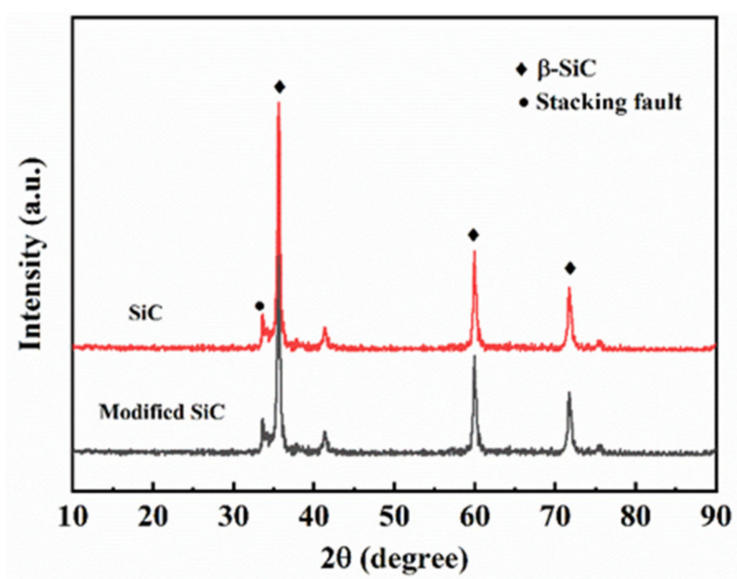

Figure 2. X-ray diffraction (XRD) patterns of the silicon carbide (SiC) powder and PVP-modified $\mathrm{SiC}$ powder.
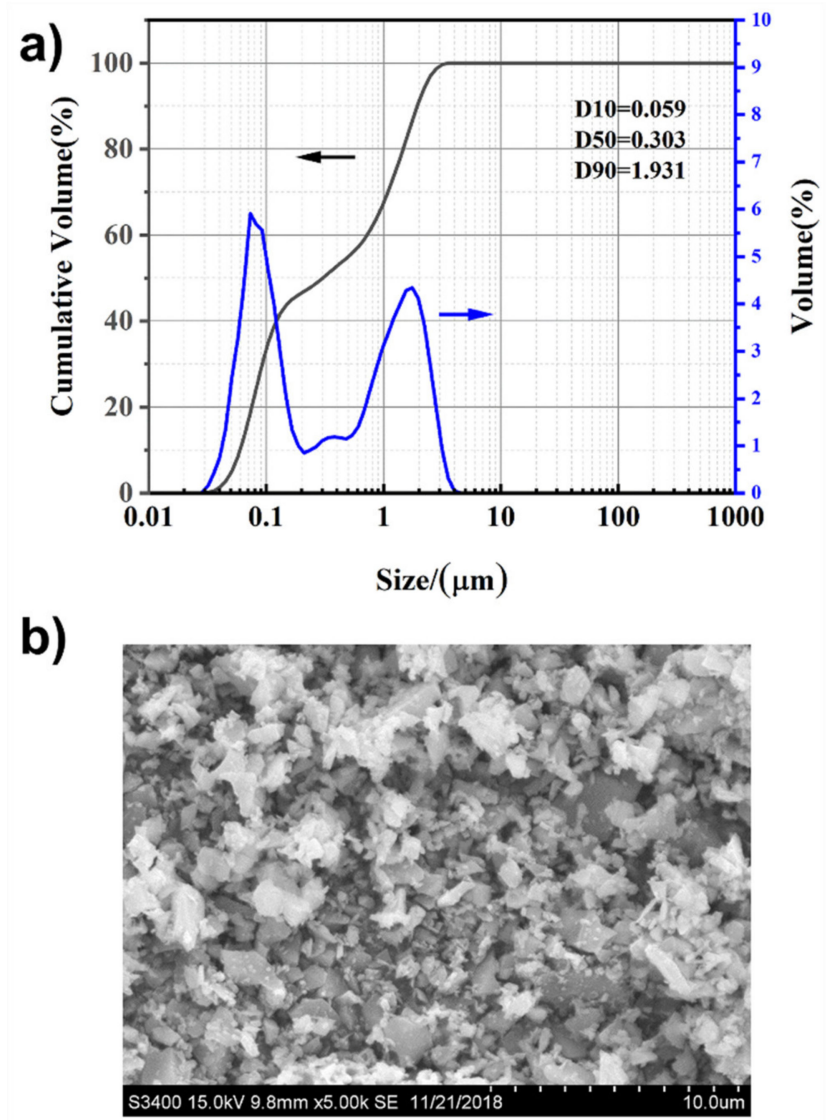

Figure 3. Particle size distribution of the as-received $\mathrm{SiC}$ powder (a) and scanning electron microscopy (SEM) image of the as-received $\mathrm{SiC}$ powder $(\mathbf{b})$.

\subsection{Rheological Measurement}

The rheological behaviors of the suspensions by adding different concentrations of dispersant were studied at a constant solids content of 20 vol.\%. The main focus was two properties: dynamic viscosity and fluid form. In Figure $4 \mathrm{a}-\mathrm{c}$, under different dispersants, the shear viscosity was plotted as a function of shear rate. As expected, the suspension at $20 \mathrm{vol} . \%$ had a very high shear viscosity. This was obviously the result of its high solids content, leading to a large increase in particle-particle interaction that gave the slurry a higher yield stress value. Figure $4 \mathrm{a}$ shows that, after the addition of 
PVP, the viscosity of the suspension decreased with an increasing concentration of added PVP, because the saturated amount of PVP was high. Thus, until the addition of PVP reached 5 wt. $\%$, saturated adsorption was achieved. A dispersant exhibits good dispersion when the dispersion has quite low viscosity at the saturated adsorption content. Figure $4 \mathrm{a}, \mathrm{b}$ show that the viscosities of the dispersions vary, and the dispersion with an addition of $5 \mathrm{wt}$.\% PVP can obtain the lowest viscosity $(17 \mathrm{mPa} \cdot \mathrm{s}$ at $\left.25 \mathrm{~s}^{-1}\right)$, while the addition of $1 \mathrm{wt}$.\% PEI can obtain a low viscosity ( $27 \mathrm{mPa} \cdot \mathrm{s}$ at $\left.25 \mathrm{~s}^{-1}\right)$. However, it is worth noting that a suspension with added PEI decreases the viscosity of the suspension as the amount of PEI increases. This was probably because the saturated adsorption amount of the PEI molecules was low; therefore, adding too much PEI increased the viscosity owing to polymer chain bridging. Figure 4c confirms that TMAH was not an appropriate additive for dispersing SiC powder in ethanol. This was because of the difficulty in ionizing in ethanol, and it was not effective to achieve electrostatic repulsion stability [25]. The data in Figure $4 \mathrm{~d}$ show the relative efficiencies of these dispersants at the determined shear rate $\left(10 \mathrm{~s}^{-1}\right)$; PVP and PEI can be identified as the efficient dispersant dispersing nano-SiC in ethanol solvent.
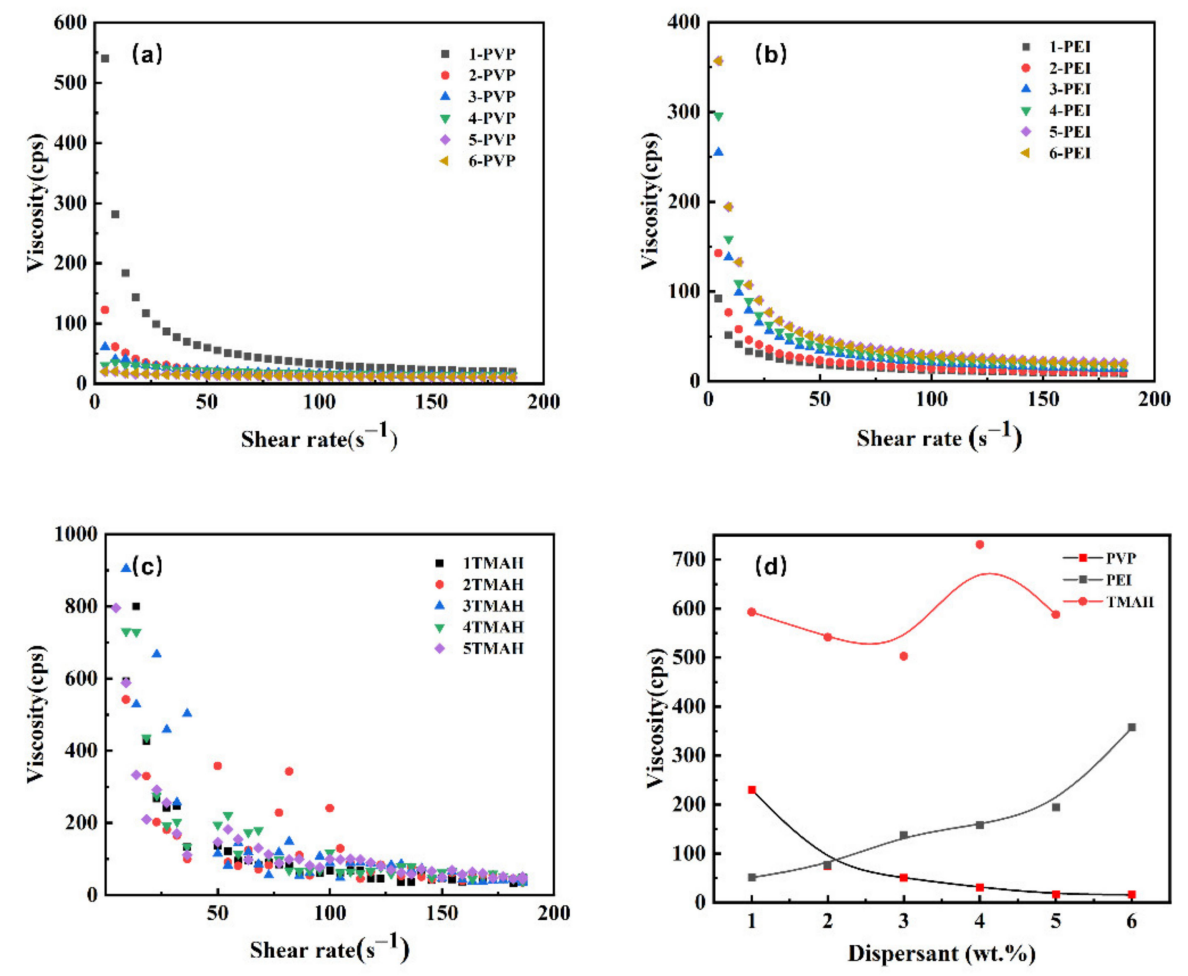

Figure 4. Viscosity as a function of the shear rate of $\mathrm{SiC}$ slurries with 20 vol.\% solids loading under different concentrations of dispersants $(\mathbf{a}-\mathbf{c})$, and the viscosity of different dispersants at the determined shear rate with $10 \mathrm{~s}^{-1}(\mathbf{d})$.

As slurry with the lowest viscosity was obtained by using PVP in the experiments, in the next step, we mainly chose PVP as a dispersant to study its effect on the flow behavior of the SiC suspensions. The rheological behavior of the dispersion can be derived from the relationship between applied shear stress and shear rate [38-41]. The relation between shear stress and shear rate can be described by a power-law relation (Equation (2)), while Equation (3) was obtained by taking the logarithm of both sides of Equation (2) [42,43].

$$
\begin{gathered}
\tau=b \dot{\gamma}^{n} \\
\log \tau=\log b+n \log \dot{\gamma} \mathrm{a}=1,
\end{gathered}
$$

where $\tau$ is the shear stress; $\dot{\gamma}$ is the applied shear rate; and $\mathrm{b}$ and $\mathrm{n}$ are the parameter and the power-law index constants, respectively. With $n<1$, the suspension was described as a shear-thinning fluid; 
when $n=1$, a Newtonian-like fluid was described; and when $n>1$, shear-thickening was described. We intensively studied the effect of PVP as a dispersant on the flow index of the suspension. The flow index was the slope of the above relation (Equation (2)) in a logarithmic plot. Figure 5 shows the variation of the flow index of the $20 \mathrm{vol}$ \% silicon carbide suspension with the increase of additional PVP (see Figure 5a-f). The suspension had multi-flow index at low amounts of dispersant (see Figure 5 $a, b)$, indicating that the shear thinning effect of the suspension under external force was obvious. In addition, as the applied shear rate increased, the flow index gradually increased. However, when the dispersant content was greater than 3wt.\%, the system exhibited a single flow index over the entire shear rate range of the experiment (see Figure $5 c-f$ ), indicating that the system exhibited stable rheological behavior. In particular, when the value of the flow index was close to 1 , it reflected quasi-Newtonian flow behavior [44]. When the appropriate amount of PVP was added, the suspension had a single flow factor with the changed shear rate, and the value approached 1 . This indicated that the suspension was in the form of a stable quasi-Newtonian fluid, compared with a suspension with a multi-flow index. Thus, PVP with an optimum dose could produce a stable colloidal dispersion, which could be reflected by a unique slope profile and quasi-Newtonian properties, as shown in Figure $5 \mathrm{e}, \mathrm{f}$, and was determined by a flow factor value approaching 1 . Regarding PVP, the literature reports that the carbonyl of PVP was beneficial for adsorbing on alkaline surfaces. Therefore, PVP was probably adsorbed via a perpendicular conformation with pyrrolidone as the adsorbing head, and the hydrogen atoms in the chain promoted absorption on the acidic ceramic surface [45-47].
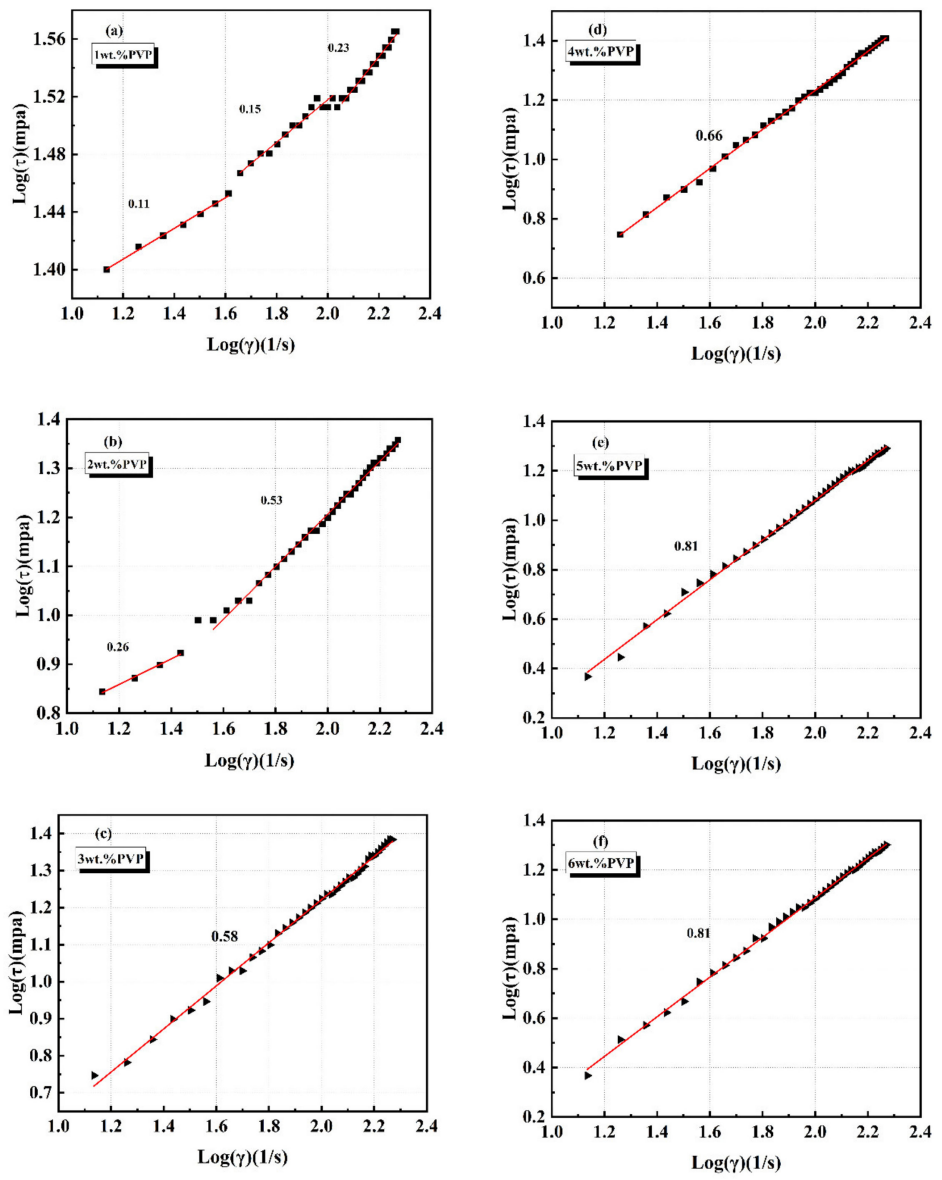

Figure 5. Double logarithmic power law plot at (a)1wt.\%, (b) 2wt.\%, (c) 3wt.\%, (d) 4wt.\%, (e) 5wt.\%, (f) $6 \mathrm{wt} . \%$ of dispersant for PVP-stabilized suspensions (at $20 \mathrm{vol} . \%$ ). The viscosity data are presented in the inset of main figure. 


\subsection{Adsorption Mechanism of Dispersants on SiC Powder}

Different solid-adsorbate systems gave different adsorption models. Adsorption models have monolayer and multilayer adsorption. The Langmuir isotherm model is the most common single-layer adsorption equation. The single-layer isotherm curve can be expressed as follows [48]:

$$
q_{\mathrm{e}}=\frac{q_{\mathrm{m}} b \mathrm{C}_{\mathrm{e}}}{\left(1+b C_{\mathrm{e}}\right)}
$$

A plot of $C_{\mathrm{e}} / q_{\mathrm{e}}$ versus $C_{\mathrm{e}}$ gives a straight line written as follows:

$$
\frac{C_{\mathrm{e}}}{q_{\mathrm{e}}}=\frac{1}{b q_{\mathrm{m}}}+\frac{C_{\mathrm{e}}}{q_{\mathrm{m}}}
$$

where $q_{\mathrm{e}}(\mathrm{mg} / \mathrm{g})$ is the balanced adsorption content, $\mathrm{b}$ is a constant, $q_{\mathrm{m}}$ is the adsorption limit, and $C_{\mathrm{e}}$ $(\mathrm{g} / \mathrm{L})$ is the amount of PVP at saturation. The Freundlich isotherm curve is a classical multilayer adsorption equation that can be expressed as follows [48]:

$$
q_{\mathrm{e}}=b C_{\mathrm{e}}^{k}
$$

Equation (7) can be obtained by taking the logarithm on both sides of Equation (6):

$$
\ln q_{\mathrm{e}}=k \ln C_{\mathrm{e}}+\ln b
$$

where $q_{\mathrm{e}}(\mathrm{mg} / \mathrm{g})$ is the equilibrium adsorption capacity, $C_{\mathrm{e}}(\mathrm{g} / \mathrm{L})$ is the concentration of the solute at equilibrium, $\mathrm{b}$ is the Freundlich parameter, and $\mathrm{k}$ is the adsorption constant. Figure 6 shows the fitted curves of the Langmuir and Freundlich models. As shown in Figure 6b, the curve obtained from the experimental data deviated from the curve fitted by the Freundlich adsorption model. Figure 6a shows that the curve obtained from the experimental data conformed to the curve plotted by the Langmuir model, which confirms that the adsorbed PVP molecule interacted with only one site and did not interact with other adsorbed molecules and formed a monolayer to some extent. The above results were also consistent with the viscosity results in Figure 4a.
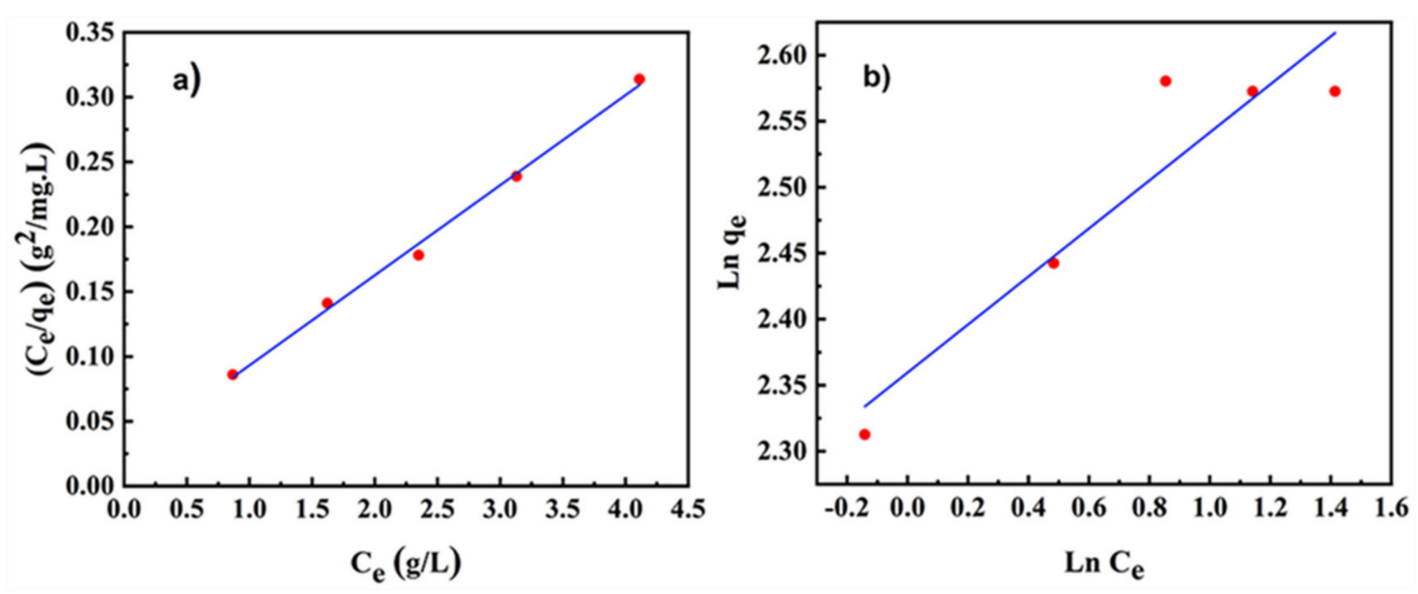

Figure 6. Langmuir model fitting (a) and Freundlich model fitting (b) of the adsorption of PVP on SiC.

\subsection{FTIR and XPS Characterization}

We further studied the chemical information of PVP molecules on the SiC surfaces. Figure 7 shows the interaction between SiC and PVP by FTIR spectroscopy. The infrared absorption band of $\mathrm{SiC}$ in Figure 7 had peak values of 3430, 1630, 943, and $835 \mathrm{~cm}^{-1}$, which were caused by $\mathrm{OH}$ stretching, $\mathrm{OH}$ stretching from water vapor by physical adsorption, $\mathrm{Si}-\mathrm{O}-\mathrm{Si}$, and $\mathrm{Si}-\mathrm{C}$ stretching 
vibration, respectively $[45,46,49]$. IR absorptions of SiC with adsorbed PVP primarily occurred at $3430,1660-1289$, and $835 \mathrm{~cm}^{-1}$. The peaks at $1630-1289 \mathrm{~cm}^{-1}$ originated from the adsorbed PVP and the $1660-1289 \mathrm{~cm}^{-1}$ peaks that emerged in the spectrum of PVP-modified $\mathrm{SiC}$ were attributed to the stretching of $\mathrm{C}-\mathrm{N}$ and $\mathrm{C}=\mathrm{O}$. These peaks confirmed that PVP was successfully adsorbed upon the $\mathrm{SiC}$ surface $[45,46]$.

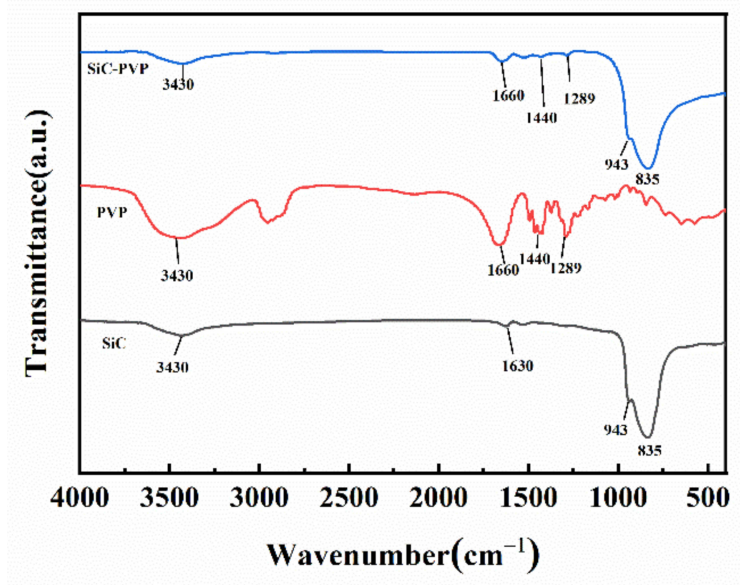

Figure 7. Fourier transform infrared spectroscopy (FTIR) spectra of SiC, PVP, and PVP-adsorbed SiC.

The chemical properties of the PVP-modified SiC were further investigated by XPS characterization. It can be seen from the data in Figure 8 that the XPS spectra of the SiC powder and modified $\mathrm{SiC}$ powder that the XPS spectra exhibited the peaks of C KLL, O KLL, C 1s, O 1s, O 2s, N 1s, Si 2s, and Si 2p. Nitrogen, as the unique element of PVP, existed on the SiC powder surface, demonstrating that PVP had been adsorbed on the SiC surface. Figure 9a,b show the C 1s spectra of PVP-modified SiC powder and unmodified $\mathrm{SiC}$ powder. The peak appearing at $290.2 \mathrm{eV}$ corresponded to $\mathrm{C}=\mathrm{O}$, which further indicated that PVP had been grafted onto the SiC surface compared with that of the pure silicon carbide powder. Figure $9 \mathrm{c}$ shows the $\mathrm{Si} 2 \mathrm{p}$ spectra of PVP-modified SiC particles. From Figure $9 \mathrm{c}$ above, we can see that the two peaks at $103.18 \mathrm{eV}$ and $104.38 \mathrm{eV}$ were related to $\mathrm{Si}-\mathrm{C}$ and $\mathrm{Si}-\mathrm{O}$, respectively [49]. The existence of $\mathrm{Si}-\mathrm{OH}$ confirmed the presence of an active site for the adsorption of PVP on the surface of silicon carbide, and hydrogen bonds could be formed between the surface of the $\mathrm{SiC}$ and PVP molecules.

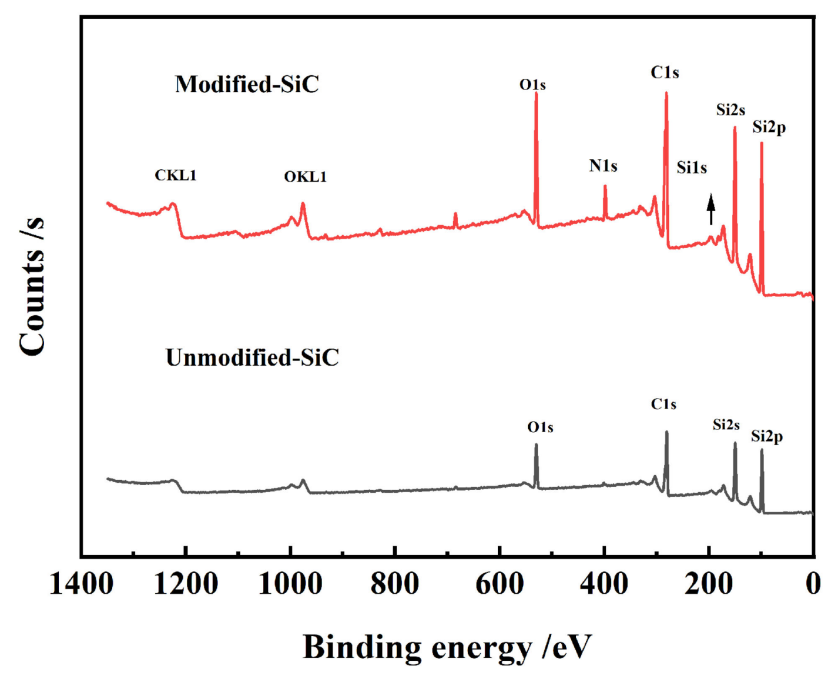

Figure 8. X-ray photoelectron spectroscopy (XPS) spectra of as-received SiC and PVP-adsorbed $\mathrm{SiC}$ particles. 

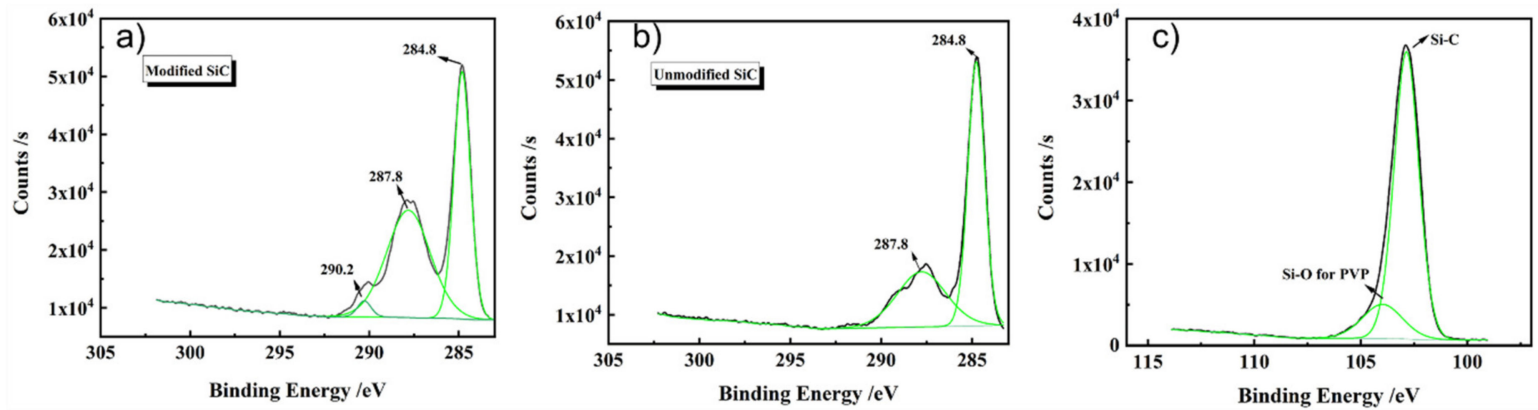

Figure 9. High-resolution XPS spectra: (a) C 1s of PVP-adsorbed SiC powder, (b) C 1s of unmodified $\mathrm{SiC}$ powder, and (c) Si $2 \mathrm{p}$ of PVP-adsorbed SiC powder.

\subsection{Maximum Ceramic Loading of the PVP Stabilized Systems}

The rheological behavior of the ceramic suspension is an important aspect of colloidal ceramic formation. Figure 10 shows suspensions with an optimal amount (5 wt.\%) of dispersant in suspension produced increased ceramic content, and its flow behaviors were studied. After the volume content of the suspensions exceeded $32 \mathrm{vol} \%$, the single flow index factor of the suspensions had a multi flow index, indicating that a soft agglomeration structure began to form inside the suspension, which was easily affected by the leading role of the applied shear force. Furthermore, we can see from Figure 11 that, when the volume loading was greater than 22.27 vol.\%, the flow index of the suspension began to mutate and the suspension began to change from a Newtonian-like fluid to a shear-thinning fluid. The flow factor method showed that PVP was a dispersant that can simultaneously produce a wide single flow index and a quasi-Newtonian region, confirming that a PVP-adsorbed SiC suspension has excellent rheological properties. Finally, we explored the issue of the maximum solids loading of the suspensions with PVP as the dispersant. Two models were used for the estimation [50]:

$$
\begin{gathered}
\frac{\eta_{n f}}{\eta_{b f}}=1+2.5 \phi \\
\frac{\eta_{n f}}{\eta_{b f}}=\left(1-\frac{\phi}{\phi_{c}}\right)^{-[\eta] \phi_{c}}
\end{gathered}
$$

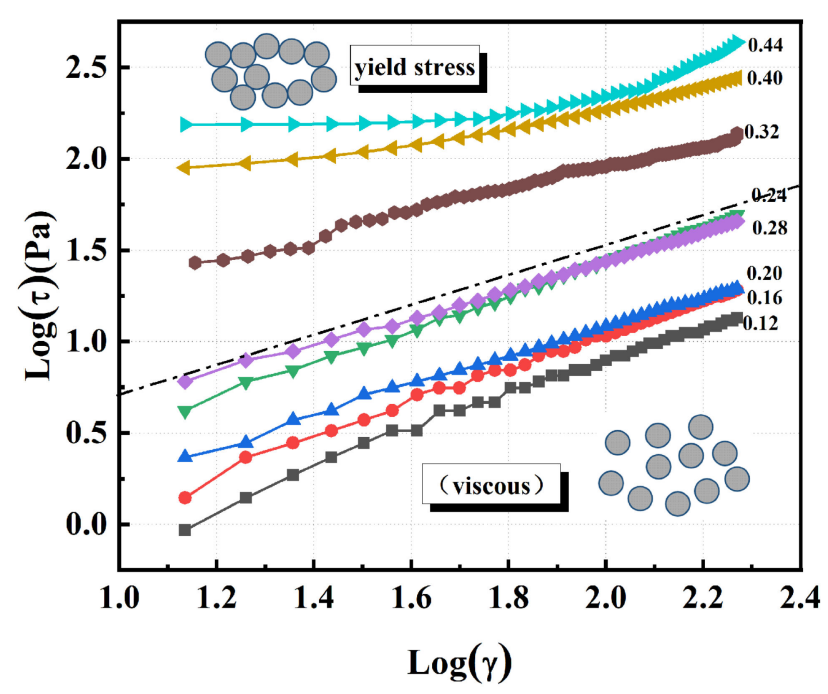

Figure 10. The logarithmic plot of the power law for $5 \mathrm{wt}$ \% PVP stabilized suspensions. The inset presents an illustration of the particle distribution model. 


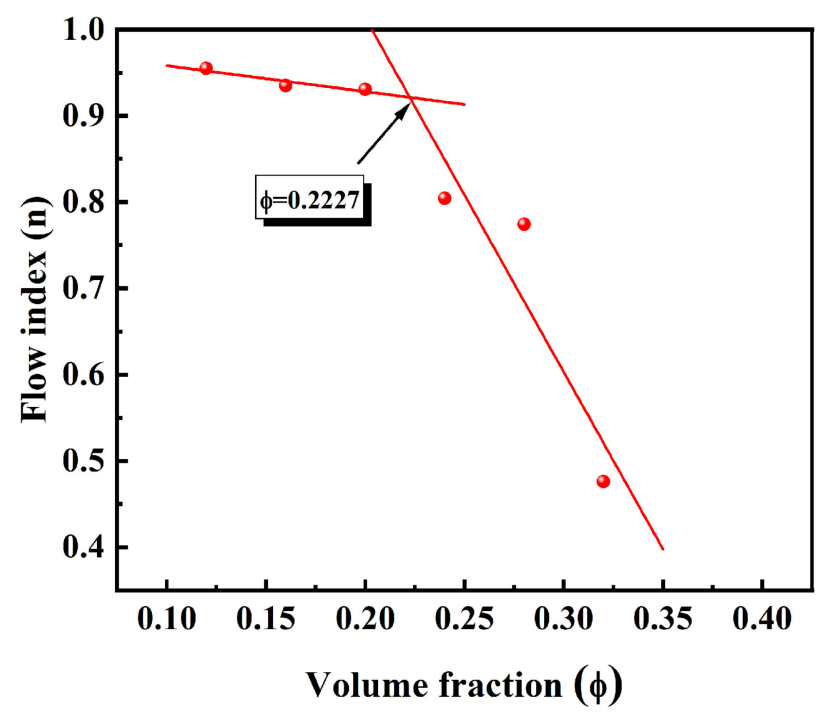

Figure 11. The plot of the flow index factor as a function of ceramic content for PVP-stabilized suspensions at an optimum PVP amount.

In the Einstein relation (Equation (8)), $\eta_{n f}$ and $\eta_{b f}$ measure the viscosity of the suspension and suspending medium, respectively, and $\phi$ is the solids loading of the suspension. The equation for the Krieger-Dougherty (K-D) model (Equation (9)) describes the loading dependence of the viscosity of suspensions, where $\phi_{c}$ is the maximum volume loading of the suspension (the viscosity of the suspension raised to infinity) and $\eta$ is the intrinsic viscosity, which could reflect the characteristics of particle agglomeration, being 2.5 for rigid particles [51]. As shown in Figure 12, we recorded the viscosity values of the suspension under different volume loadings. We also plotted the K-D model curve and the Einstein viscosity curve. The results of Figure 12 illustrate that the K-D model fitted well with the experimental data at a higher volume fraction and can be used to predict the maximum solid phase content. In contrast, the Einstein model fits well only at low concentrations. From the K-D model, we can obtain a maximum volume content of $46 \mathrm{vol} \% \mathrm{SiC}$ slurry.

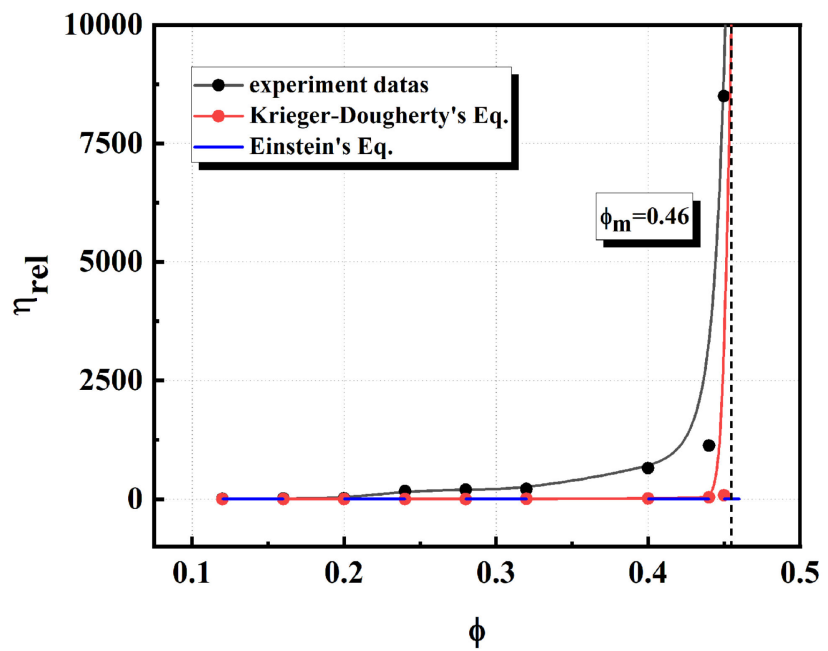

Figure 12. The relative viscosity of the SiC slurry as a function of solids loading determined by different models.

\section{Conclusions}

The main goal of the current study was to determine the optimal dispersing agents with different chemical properties to disperse silicon carbide powder in an organic solvent. The three dispersants 
were amphiphilic, acidophilic, and alkaliphilic. As a result, PVP and PEI were found to be suitable for achieving good dispersion of nano-SiC in ethanol. In addition, the rheological index factor was put forward to quantitatively characterize the rheological properties of the slurry. The single index factor reflected the good stability of the suspension. Further investigation of the adsorption mechanism was discussed.

The adsorption mechanism of PVP-modified silicon carbide powder was first investigated. The results obtained revealed that the adsorption isotherm equation was basically consistent with the Langmuir isotherm model. FTIR and XPS data indicated that PVP successfully modified the surface of the silicon carbide particles.

In the final part of the experiments, the maximum ceramic loading of PVP-stabilized systems was investigated. We found that using the K-D model to predict the maximum solids content of the slurry at high solids levels was more reliable compared with the other models. The theoretical maximum solids content of the silicon carbide suspension was $46 \mathrm{vol} \%$ with micro- and nanoparticles stabilized by PVP.

Author Contributions: Conceptualization, G.L. and Z.Z.; Methodology, Z.Z. and J.H.; Validation, Z.Z.; Investigation, Z.Z.; Writing-Original draft preparation, Z.Z.; Writing-Review and editing, Z.Z. and G.L.; Visualization, G.L.; Supervision, J.Z. and Y.S.; Project administration, Q.S.; Funding acquisition, L.Z. All authors have read and agreed to the published version of the manuscript.

Funding: This research was funded by the State key Program of National Natural Science Foundation of China (Grant No. 51932006); National Natural Science Foundation of China (Grant No. 51521001); the "111" Project (Grant No. B13035); the Joint Fund (Grant No. 6141A02022255); and the major program of the specialized technological innovation of HuBei Province, China (Grant No. 2019AFA176).

Conflicts of Interest: The authors declare no conflict of interest.

\section{References}

1. Casady, R.W.J.B. Status of silicon carbide $(\mathrm{SiC})$ as a wide bandgap semiconductor for high temperature applications: A review. J. Solid State Electron. 1996, 39, 1409-1422. [CrossRef]

2. Nakamura, D.; Gunjishima, I.; Yamaguchi, S. Ultrahigh-Quality Silicon Carbide Single Crystals. Nature 2004, 430, 1009-1012. [CrossRef] [PubMed]

3. Madar, R. Material Science-Silicon carbide in contention. Nature 2004, 430, 974-975. [CrossRef] [PubMed]

4. Lange, F.F. Powder Processing Science and Technology for Increased Reliability. J. Am. Ceram. Soc. 1989, 72, 3-15. [CrossRef]

5. Fan, J.; Chu, P.K. Silicon Carbide Nanostructures: Fabrication, Structure, and Properties; Springer International Publishing: Berlin/Heidelberg, Germany, 2014; p. 330.

6. Hotza, D.; Greil, P. Review: Aqueous tape casting of ceramic powders. Mater. Sci. Eng. A 1994, 202, $206-217$. [CrossRef]

7. Montanaro, L.; Coppola, B.; Palmero, P. A review on aqueous gelcasting: A versatile and low-toxic technique to shape ceramics. Ceram. Int. 2019, 45, 9653-9673. [CrossRef]

8. Jabbari, M.; Bulatova, R.; Tok, A.I.Y. Ceramic tape casting: A review of current methods and trends with emphasis on rheological behaviour and flow analysis. Mater. Sci. Eng. B 2016, 212, 39-61. [CrossRef]

9. Yu, J.; Yang, J.; Huang, Y. The transformation mechanism from suspension to green body and the development of colloidal forming. Ceram. Int. 2011, 37, 1435-1451. [CrossRef]

10. Iijima, M. Surface modification techniques toward controlling the dispersion stability and particle-assembled structures of slurries. J. Ceram. Soc. Jpn. 2017, 125, 603-607. [CrossRef]

11. Rödel, C.; Müller, M.; Glorius, M. Effect of varied powder processing routes on the stabilizing performance and coordination type of polyacrylate in alumina suspensions. J. Eur. Ceram. Soc. 2011, 32, 363-370. [CrossRef]

12. Zhang, Y.; Binner, J. Effect of dispersants on the rheology of aqueous silicon carbide suspensions. Ceram. Int. 2008, 34, 1381-1386. [CrossRef]

13. Makio Naito, T.Y.K.H. Nanoparticle Technology Handbook; Elsevier: Amsterdam, The Netherlands, 2018. 
14. Ganguly, S.; Chakraborty, S. Sedimentation of nanoparticles in nanoscale colloidal suspensions. Phys. Lett. A 2011, 375, 2394-2399. [CrossRef]

15. Vázquez, G.; Alvarez, E.; Navaza, J.M. Surface Tension of Alcohol + Water from 20 to $50{ }^{\circ}$ C. J. Chem. Eng. Data. 1995, 40, 611-614. [CrossRef]

16. Raiteri, P.; Laio, A.; Parrinello, M. Correlations among hydrogen bonds in liquid water. Phys. Rev. Lett. 2004, 93, 87801. [CrossRef] [PubMed]

17. Luzar, A.; Chandler, D. Hydrogen-bond kinetics in liquid water. Nature 1996, 379, 55-57. [CrossRef]

18. Riedel, R.; Chen, I.W. Ceramics Science and Technology: Volume3: Synthesis and Processing; Wiley-VCH: Weinheim, Germany, 2011.

19. Chin, C.H.; Muchtar, A.; Azhari, C.H.; Razali, M. Optimization of pH and Dispersant Amount of Y-TZP Suspension for Colloidal Stability. Ceram Int. 2015, 41, 9939-9946. [CrossRef]

20. Li, C.; Liu, W.; Chen, Y. Efficient dispersants for the dispersion of gallium zinc oxide nanopowder in aqueous suspensions. J. Am. Ceram. Soc. 2017, 100, 920-928. [CrossRef]

21. Foratirad, H.; Baharvandi, H.R.; Maragheh, M.G. Effects of dispersants on dispersibility of titanium carbide aqueous suspension. Int. J. Refract. Met. H 2016, 56, 96-103. [CrossRef]

22. Zhang, J.; Xu, Q.; Ye, F. Effect of citric acid on the adsorption behavior of polyethylene imine (PEI) and the relevant stability of SiC slurries. Colloids Surf. A 2006, 276, 168-175. [CrossRef]

23. Avadiar, L.; Leong, Y. Interactions of PEI (polyethylenimine)-silica particles with citric acid in dispersions. Colloid. Poly. Sci. 2011, 289, 237-245. [CrossRef]

24. Barick, P.; Prasad, S.B.; Mitra, R. Effect of concentration and molecular weight of polyethylenimine on zeta potential, isoelectric point of nanocrystalline silicon carbide in aqueous and ethanol medium. Ceram. Int. 2015, 41, 4289-4293. [CrossRef]

25. Li, W.; Chen, P.; Gu, M. Effect of TMAH on rheological behavior of SiC aqueous suspension. J. Eur. Ceram. Soc. 2004, 24, 3679-3684. [CrossRef]

26. Iijima, M.; Okamura, N.; Tatami, J. Polyethyleneimine-Oleic Acid Complex as a Polymeric Dispersant for Si3N4 and Si3N4-Based Multicomponent Nonaqueous Slurries. Ind. Eng. Chem. Res. 2015, 54, 12847-12854. [CrossRef]

27. Li, C.; Jhang, J.; Tsai, H. Water-soluble polyethylenimine as an efficient dispersant for gallium zinc oxide nanopowder in organic-based suspensions. Powder. Technol. 2017, 305, 226-231. [CrossRef]

28. Liu, Y.; Liu, J. Study on modification effect and mechanism of binary modifier co-modified silicon carbide powder. Mater. Res. Express. 2018, 6, 35204. [CrossRef]

29. Zhang, J.; Sun, Y.; Shimai, S. Effect of TMAH on the rheological behavior of alumina slurries for gelcasting. J. Asian. Ceram. Soc. 2018, 5, 261-265. [CrossRef]

30. Vieira, L.E.; Neto, J.B.R.; Klein, A.N. Colloidal processing of highly concentrated aqueous copper suspensions. Powder. Technol. 2014, 256, 540-544. [CrossRef]

31. Vilinska, A.; Ponnurangam, S.; Chernyshova, I. Stabilization of Silicon Carbide (SiC) micro- and nanoparticle dispersions in the presence of concentrated electrolyte. J. Colloid. Interf. Sci. 2014, 423, 48-53. [CrossRef]

32. Gan, K.; Xu, J.; Lu, Y. Preparation of silicon carbide ceramics using chemical treated powder by DCC via dispersant reaction and liquid phase sintering. J. Eur. Ceram. Soc. 2017, 37, 891-897. [CrossRef]

33. Barick, P.; Mitra, R.; Saha, B.P. Influence of a few important parameters on the rheological behaviour of silicon carbide nanoparticles dispersed aqueous suspension. Ceram. Int. 2018, 44, 9070-9075. [CrossRef]

34. Marani, D.; Sudireddy, B.R.; Nielsen, L. Poly(vinylpyrrolidone) as dispersing agent for cerium-gadolinium oxide (CGO) suspensions. J. Mater. Sci. 2016, 51, 1098-1106. [CrossRef]

35. Bailly, N.; Thomas, M.; Klumperman, B. Poly(N-vinylpyrrolidone)-block-poly(vinyl acetate) as a Drug Delivery Vehicle for HydrophobicDrugs. Biomacromolecules 2012, 13, 4109-4117. [CrossRef]

36. Kim, D.; Park, I.; Lee, M. Tensile strength of aqueous-based alumina tapes using a PVP-PVA-gelatin cobinder. Ceram. Int. 2005, 31, 577-581. [CrossRef]

37. Ong, B.C.; Leong, Y.K.; Chen, S.B. Interparticle forces in spherical monodispersed silica dispersions: Effects of branched polyethylenimine and molecular weight. J. Colloid. Interf. Sci. 2009, 337, 24-31. [CrossRef]

38. Hasanzadeh, M.; Mottaghitalab, V.; Rezaei, M. Rheological and viscoelastic behavior of concentrated colloidal suspensions of silica nanoparticles: A response surface methodology approach. Adv. Powder. Technol. 2015, 26, 1570-1577. [CrossRef] 
39. Li, X.; Zou, C.; Wang, T. Rheological behavior of ethylene glycol-based SiC nanofluids. Int. J. Heat. Mass. Tran. 2015, 84, 925-930. [CrossRef]

40. Cwalina, C.D.; Wagner, N.J. Rheology of non-Brownian particles suspended in concentrated colloidal dispersions at low particle Reynolds number. J. Rheol. 2016, 60, 47-59. [CrossRef]

41. Jamali, S.; Boromand, A.; Wagner, N. Microstructure and rheology of soft to rigid shear-thickening colloidal suspensions. J. Rheol. 2015, 59, 1377-1395. [CrossRef]

42. Marani, D.; Hjelm, J.; Wandel, M. Rheological analysis of stabilized cerium-gadolinium oxide (CGO) dispersions. J. Eur. Ceram. Soc. 2014, 34, 695-702. [CrossRef]

43. Willenbacher, N.; Georgieva, K. Rheology of Disperse Systems; Wiley-VCH: Weinheim, Germany, 2013; pp. 7-46.

44. Xiao, C.; Gao, L.; Lu, M. Effect of polyvinylpyrrolidone on rheology of aqueous SiC suspensions with polyethylene glycol as binder. Colloids Surf. A 2010, 368, 53-57. [CrossRef]

45. Xiao, C.; Gao, L.; Lu, M. Synergistic effect of copolymer and poly(vinylpyrrolidone) mixtures on rheology of aqueous SiC suspensions. Colloids Surf. A 2010, 355, 104-108. [CrossRef]

46. De Riccardis, M.F.; Carbone, D. Study of Polymer Particles Suspensions for Electrophoretic Deposition. J. Phys. Chem. B 2013, 117, 1592-1599. [CrossRef] [PubMed]

47. Kissa, E. Dispersions: Characterization, Testing, and Measurement; Dekker, M., Ed.; CRC Press: Boca Raton, FL, USA, 1999; p. 695.

48. George, V.C.; Das, A.; Roy, M. Bias enhanced deposition of highly oriented $\beta$-SiC thin films using low pressure hot filament chemical vapour deposition technique. Thin Solid Films 2002, 410, 114-117. [CrossRef]

49. Li, S.; Wang, J.; Zhao, S. Effect of surface modification and medium on the rheological properties of silica nanoparticle suspensions. Ceram. Int. 2016, 42, 7767-7773. [CrossRef]

50. Mewis, J.; Wagner, N.J. Colloidal Suspension Rheology; Cambridge University Press: Cambridge, UK, 2012; p. 415.

51. Smith, T.L.; Bruce, C.A. Intrinsic viscosities and other rheological properties of flocculated suspensions of nonmagnetic and magnetic ferric oxides. J. Colloid Interf. Sci. 1979, 72, 13-26. [CrossRef]

(C) 2020 by the authors. Licensee MDPI, Basel, Switzerland. This article is an open access article distributed under the terms and conditions of the Creative Commons Attribution (CC BY) license (http://creativecommons.org/licenses/by/4.0/). 